\title{
Oscillation of nonlinear neutral dynamic equations on time scales
}

\author{
G. N. Chhatria', Said R. Grace ${ }^{2}$ and John R. Graef ${ }^{3 *}$ (1)
}

\section{${ }^{*}$ Correspondence:}

John-Graef@utc.edu

${ }^{3}$ Department

of Mathematics, University

of Tennessee at Chattanooga

Chattanooga, TN 37403, USA

Full list of author information

is available at the end of the

article

\begin{abstract}
The authors present necessary and sufficient conditions for the oscillation of a class of second order non-linear neutral dynamic equations with non-positive neutral coefficients by using Krasnosel'skii's fixed point theorem on time scales. The nonlinear function may be strongly sublinear or strongly superlinear.
\end{abstract}

Keywords: Oscillation, Nonoscillation, Neutral dynamic equation, Time scales, Fixed point theorem

Mathematics Subject Classification: 34C10, 34K11, 34N05, 39A 10

\section{Introduction}

Neutral differential/difference equations find numerous applications in biology, engineering, economics, physics, neural networks, social sciences, etc (see, for example, [4, $12,16])$. In the last few decades, many authors have focused their interest on the study of the oscillation of solutions of neutral differential/difference equations with deviating arguments, and in this regard, we refer the reader to the monographs of Agarwal et al. $[1,2]$ and the papers $[3,7-11,13-15,22,29]$.

Introduced by Stefan Hilger [17], the notion of time scales is not only to unify the theories of differential equations and difference equations, but also to extend some cases in between these classical ones. For details on the theory of dynamic equations on time scales and its applications as well as for basic concepts and notations, we refer the reader to the works of Bohner and Peterson [5, 6]. By employing a Riccati transformation technique and applying some inequalities, a large number of papers have been devoted to the oscillatory behavior of solutions to second order dynamic equations with nonnegative neutral coefficients; for example, see [3, 8, 9, 23-27] and the references cited therein. At the same time, there are comparatively few papers concerned with the oscillation of equations with nonpositive neutral coefficients; for example, see [7, 14, 18, 20, 28].

Bohner and Li [7] considered the second order dynamic equation

$$
\left(r(\ell)\left|z^{\Delta}(\ell)\right|^{p-2} z^{\Delta}(\ell)\right)^{\Delta}+q(\ell)|x(\delta(\ell))|^{p-2} x(\delta(\ell))=0,
$$
party material in this article are included in the article's Creative Commons licence, unless indicated otherwise in a credit line to the material. If material is not included in the article's Creative Commons licence and your intended use is not permitted by statutory regulation or exceeds the permitted use, you will need to obtain permission directly from the copyright holder. To view a copy of this licence, visit http:// creativecommons.org/licenses/by/4.0/. 
where $z(\ell)=x(\ell)-a(\ell) x(\tau(\ell)), p>1$ is a constant, and $0 \leq a(\ell) \leq a_{0}<1$. They improved the papers $[14,18]$ by developing a new method for the analysis of the oscillation of (1) via a comparison principle.

Recently, Zhang et al. [28] discussed the neutral dynamic equation

$$
\left(r(\ell)\left(z^{\Delta}(\ell)\right)^{\alpha}\right)^{\Delta}+q(\ell) f(x(\delta(\ell)))=0,
$$

where $z(\ell)=x(\ell)-p(\ell) x(\tau(\ell)), \quad \alpha \geq 1$ is a quotient of odd positive integers, $0 \leq p(\ell) \leq p_{0}<1$, and there is a positive constant $k$ such that $\frac{f(x)}{x^{\alpha}} \geq k$ for all $x \neq 0$. They present some new oscillation criteria to ensure that a solution of (2) either oscillates or converges to zero.

Motivated by the results in [28] and the discussion above, in this work we wish to find conditions that are sufficient as well as necessary for the oscillation of second order nonlinear dynamic equations on time scales of the form

$$
\left[a(\ell)\left(v^{\Delta}(\ell)\right)^{\alpha}\right]^{\Delta}+\Lambda(\ell) u^{\beta}(\tau(\ell))=0, \quad \ell \in\left[\ell_{0}, \infty\right)_{\mathbb{T}},
$$

where the time scale $\mathbb{T}$ satisfies $\sup \mathbb{T}=\infty, \quad \ell \in\left[\ell_{0}, \infty\right)_{\mathbb{T}}$ with $\ell_{0} \in \mathbb{T}$, and $v(\ell)=u(\ell)+q(\ell) u(m(\ell))$. A solution of (3) is a real function $u \in C_{r d}^{1}\left[\ell_{0}, \infty\right)_{\mathbb{T}}$ such that $a(\ell)\left(v^{\Delta}(\ell)\right)^{\alpha} \in C_{r d}^{1}\left[\ell_{0}, \infty\right)_{\mathbb{T}}$ and which satisfies $(3)$ on $\left[T_{u}, \infty\right)_{\mathbb{T}}$, where $T_{u}>\ell_{0}$ is chosen so that $\tau(\ell)>\ell_{0}$ for $\ell \geq T_{u}$, and $C_{r d}(\mathbb{T}, \mathbb{R})$ is the space of real valued right-dense continuous functions (see [5]). Throughout this paper, we restrict our attention to those solutions of (3) that exist on some half line $\left[\ell_{u}, \infty\right)_{\mathbb{T}}$ and satisfy $\sup \{|u(\ell)|: \ell \geq T\}>0$ for any $T>T_{u}$. Such a solution is said to be oscillatory if it is not eventually positive or eventually negative, and to be nonoscillatory otherwise.

Throughout, we assume that:

$\left(\mathcal{H}_{1}\right) \quad \alpha, \beta$ are quotient of odd positive integers, $\alpha>1$, and $-1<q_{1} \leq q(\ell) \leq 0$;

$\left(\mathcal{H}_{2}\right) \quad m, \quad \tau \in C_{r d}\left(\left[l_{0}, \infty\right)_{\mathbb{T}}, \mathbb{T}\right) \quad$ with $\quad m(\ell) \leq \ell, \quad \tau(\ell) \leq \ell, \quad$ and $\lim _{\ell \rightarrow \infty} m(\ell)=\lim _{\ell \rightarrow \infty} \tau(\ell)=\infty$;

$\left(\mathcal{H}_{3}\right) \quad \Lambda, a \in C_{r d}\left(\left[\ell_{0}, \infty\right)_{\mathbb{T}}, \mathbb{R}_{+}\right)$with $\Lambda(\ell) \not \equiv 0$ and

$$
\int_{\ell_{0}}^{\infty} \frac{\Delta s}{a^{1 / \alpha}(s)}=\infty
$$

Defining

$$
\mathcal{A}(\ell)=\int_{\ell_{0}}^{\ell} \frac{\Delta s}{a^{1 / \alpha}(s)}
$$

we have $\lim _{\ell \rightarrow \infty} \mathcal{A}(\ell)=\infty$.

\section{Methods}

The approach used involves the construction of an appropriate Banach space and defining two mappings. The sum of these two mapping then yields an operator that is equivalent to an integral representation of the solution to the nonlinear dynamic equation (3) under investigation. By applying Krasnosel'skii's fixed point theorem on time scales, it is then possible to obtain a fixed point of the operator that in turn 
corresponds to a solution of Eq. (3). Once this is accomplished, various qualitative properties of solution can be obtained.

\section{Results}

In what follows, all functional inequalities are assumed to hold eventually, that is, they are satisfied for all $\ell$ large enough. Without loss of generality, in our proofs we only deal with positive solutions of (3).

The following two lemmas provide some inequalities that will be useful in our proofs.

Lemma 1 Let $0<\omega<1$ be the ratio of odd positive integers and $A, B \geq 0$ with $A \geq B$. Then: $A^{\omega}-B^{\omega} \leq(A-B)^{\omega}$.

Proof For $x \geq 1$ let $f(x)=(x-1)^{\omega}-x^{\omega}+1$. Then,

$$
f^{\prime}(x)=\omega\left[(x-1)^{\omega-1}-x^{\omega-1}\right]=\left[\frac{x^{1-\omega}-(x-1)^{1-\omega}}{x^{1-\omega}(x-1)^{1-\omega}}\right] \geq 0
$$

for $x>1$. Therefore, $f(x) \geq f(1)=0$ for $x \geq 1$. Letting $x=A / B$ proves the lemma.

Lemma 2 [15] Suppose that $\omega>0$ and $|x|^{\Delta}$ is of one sign on $\left[t_{0}, \infty\right)$. Then

$$
\frac{|x|^{\Delta}}{\left(|x|^{\sigma}\right)^{\omega}} \leq \frac{\left(|x|^{1-\omega}\right)^{\Delta}}{1-\omega} \leq \frac{|x|^{\Delta}}{|x|^{\omega}} \text { on }\left[t_{0}, \infty\right)
$$

Lemma 3 below can be proved by following the lines of the proof of [20, Lemma 2.1].

Lemma 3 Let $u$ be an eventually positive solution of (3). Then $v$ satisfies one of the following cases:

(a) $\quad v>0, v^{\Delta}>0$, and $\left(a\left(v^{\Delta}\right)^{\alpha}\right)^{\Delta} \leq 0$;

(b) $v<0, v^{\Delta}>0$, and $\left(a\left(v^{\Delta}\right)^{\alpha}\right)^{\Delta} \leq 0$

for $\ell \in \mathbb{T}$ sufficiently large.

Lemma 4 Let $u$ be an eventually positive solution of (3) such that $v$ satisfies case (b) of Lemma 3. Then

$$
\lim _{\ell \rightarrow \infty} u(\ell)=0
$$

Proof Let $u$ be an eventually positive solution of (3) with $u(m(\ell))>0$ and $u(\tau(\ell))>0$ and such that Lemma 3(b) holds for $\ell \geq \ell_{1}$ for some $\ell_{1} \geq \ell_{0}$. Then $v(\ell)<0$ and $v^{\Delta}(\ell)>0$ for $\ell \geq \ell_{1}$, so $v(\ell)$ is bounded.

We will consider two possibilities. First assume that $u(\ell)$ is bounded. Then,

$$
\limsup _{\ell \rightarrow \infty} u(\ell)=L \quad \text { with } \quad 0 \leq L<\infty .
$$


To show that $L=0$, assume that $L>0$. Then there is a sequence $\left\{\ell_{k}\right\} \rightarrow \infty$ such that $\left\{u\left(\ell_{k}\right)\right\} \rightarrow L$ as $\ell \rightarrow \infty$. Let $\epsilon=-L\left(1+q_{1}\right) / 2 q_{1}>0$; then for large $k, u\left(m\left(\ell_{k}\right)\right)<L+\epsilon$, so

$$
0 \geq \lim _{k \rightarrow \infty} v\left(\ell_{k}\right)=\lim _{k \rightarrow \infty}\left[u\left(\ell_{k}\right)+q\left(\ell_{k}\right) u\left(m\left(\ell_{k}\right)\right)>L+q_{1}(L+\epsilon)>L\left(1+q_{1}\right) / 2>0,\right.
$$

which is a contradiction.

Finally, to complete the proof, we need to show that $u(\ell)$ is not unbounded. If $u(\ell)$ is unbounded, then there is a sequence $\left\{\ell_{j}\right\} \rightarrow \infty$ such that $\left\{u\left(\ell_{j}\right)\right\} \rightarrow \infty$ as $j \rightarrow \infty$ and $u\left(\ell_{j}\right)=\max \left\{u(\ell): \ell_{0} \leq \ell \leq \ell_{j}\right\}$. Now $\left\{m\left(\ell_{j}\right)\right\} \rightarrow \infty$ and $m\left(\ell_{j}\right) \leq \ell_{j}$, so

$$
u\left(m\left(\ell_{j}\right)\right) \leq \max \left\{u(\ell): \ell_{0} \leq \ell \leq \ell_{j}\right\}=u\left(\ell_{j}\right) .
$$

Hence, for large $j$,

$$
v\left(\ell_{j}\right)=u\left(\ell_{j}\right)+q\left(\ell_{j}\right) u\left(m\left(\ell_{j}\right)\right) \geq u\left(\ell_{j}\right)+q_{1} u\left(m\left(\ell_{j}\right)\right) \geq\left(1+q_{1}\right) u\left(\ell_{j}\right)>0,
$$

which contradicts the fact that $v(\ell)<0$. This completes the proof of the lemma.

Our first result on the asymptotic behavior of solutions of Eq. (3) is as follows.

Theorem 5 Let $\left(\mathcal{H}_{1}\right)-\left(\mathcal{H}_{3}\right)$ hold and assume that $\alpha \geq 1$ and there is a constant $\gamma \in \mathbb{R}_{+}$such that $\beta<\gamma<\alpha$. Then any solution of (3) either oscillates or satisfies $\lim _{\ell \rightarrow \infty} u(\ell)=0$ if and only if

$\left(\mathcal{H}_{4}\right) \quad \int_{\ell_{0}}^{\infty} \Lambda(s) \mathcal{A}^{\beta}(\tau(s)) \Delta s=\infty$.

Proof Necessity: To prove the necessity of the condition, assume that $\left(\mathcal{H}_{4}\right)$ does not hold. Then there exists $\ell_{1}>\ell_{0}$ such that

$$
\left.\int_{\ell_{1}}^{\infty} \Lambda(s) \mathcal{A}^{\beta}(\tau(s))\right) \Delta s<\infty
$$

Let

$$
\chi=\left\{u: u \in C_{r d}\left(\left[\ell_{0}, \infty\right)_{\mathbb{T}}, \mathbb{R}\right) \mid \sup _{\ell \in\left[\ell_{0}, \infty\right)_{\mathbb{T}}} \frac{u(\ell)}{\mathcal{A}(\ell)}<\infty\right\} .
$$

Clearly, $\chi$ is a Banach space with the norm $\|u\|=\sup _{\ell \in\left[\ell_{0}, \infty\right)_{\mathbb{T}}} \frac{u(\ell)}{\mathcal{A}(\ell)}$. For any $\varsigma_{1}>0$, $\varsigma_{2}>0$, and $\ell^{*} \in\left[\ell_{0}, \infty\right)_{\mathbb{T}}$ with $\varsigma_{1}<\left(1+q_{1}\right)_{\varsigma_{2}}$, let $\Omega_{\varsigma_{1}, \varsigma_{2}} \subset \chi$ be given by

$$
\Omega_{\varsigma_{1}, \varsigma_{2}}=\left\{u \in \chi: \varsigma_{1}\left[\mathcal{A}(\ell)-\mathcal{A}\left(\ell^{*}\right)\right] \leq u(\ell) \leq \varsigma_{2}\left[\mathcal{A}(\ell)-\mathcal{A}\left(\ell^{*}\right)\right], \ell \in\left[\ell_{0}, \infty\right)_{\mathbb{T}}\right\} .
$$

By (4), we can find $\ell^{*}>\ell_{1}, \varsigma_{1}, \varsigma_{2}$, and $\varsigma_{3}$ such that $\left(\varsigma_{1}\right)^{\alpha}<\varsigma_{3}<\left(\left(1+q_{1}\right) \varsigma_{2}\right)^{\alpha}$ and

$$
\left.\int_{\ell^{*}}^{\infty} \Lambda(s) \mathcal{A}^{\beta}(\tau(s))\right) \Delta s \leq \frac{\left(\left(1+q_{1}\right) \varsigma_{2}\right)^{\alpha}-\varsigma_{3}}{\varsigma_{2}^{\beta}} .
$$

Define two maps $\Gamma_{1}$ and $\Gamma_{2}$ on $\Omega_{\varsigma_{1}, \varsigma_{2}}$ by 


$$
\left(\Gamma_{1} u\right)(\ell)= \begin{cases}\left(\Gamma_{1} u\right)\left(\ell^{*}\right), & \ell \in\left[\ell_{0}, \ell^{*}\right)_{\mathbb{T}} \\ -q(\ell) u(m(\ell)), & \ell \in\left[\ell^{*}, \infty\right)_{\mathbb{T}}\end{cases}
$$

and

$$
\left(\Gamma_{2} u\right)(\ell)= \begin{cases}\left(\Gamma_{2} u\right)\left(\ell^{*}\right), & \ell \in\left[\ell_{0}, \ell^{*}\right)_{\mathbb{T}} \\ \int_{\ell^{*}}^{\ell}\left[\frac{1}{a(s)}\left[\varsigma_{3}+\int_{s}^{\infty} \Lambda(\theta) u^{\beta}(\tau(\theta)) \Delta \theta\right]\right]^{1 / \alpha} \Delta s, & \ell \in\left[\ell^{*}, \infty\right)_{\mathbb{T}}\end{cases}
$$

First, we show that for any $u_{1}, u_{2} \in \Omega_{\varsigma_{1}, \zeta_{2}}$, we have $\Gamma_{1} u_{1}+\Gamma_{2} u_{2} \in \Omega_{\varsigma_{1}, \zeta_{2}}$. To do this, let $u_{1}, u_{2} \in \Omega_{\varsigma_{1}, \varsigma_{2}}$. Note that $u(\ell) \leq \varsigma_{2} \mathcal{A}(\ell)$, so $u^{\beta}(\tau(\ell)) \leq \varsigma_{2}^{\beta} \mathcal{A}^{\beta}(\tau(\ell))$. This, together with (5) implies that for $\ell \geq \ell^{*}$,

$$
\begin{aligned}
\left(\Gamma_{1} u_{1}\right)(\ell)+\left(\Gamma_{2} u_{2}\right)(\ell) & =-q(\ell) u_{1}(m(\ell))+\int_{\ell^{*}}^{\ell}\left[\frac{1}{a(s)}\left[\varsigma_{3}+\int_{s}^{\infty} \Lambda(\theta) u_{2}^{\beta}(\tau(\theta)) \Delta \theta\right]\right]^{1 / \alpha} \Delta s \\
& \leq-q(\ell) u_{1}(m(\ell))+\int_{\ell^{*}}^{\ell}\left[\frac{1}{a(s)}\left[\varsigma_{3}+\int_{s}^{\infty} \varsigma_{2}^{\beta} \Lambda(\theta) \mathcal{A}^{\beta}(\tau(\theta)) \Delta \theta\right]\right]^{1 / \alpha} \Delta s \\
& \leq-q_{1} \varsigma_{2}\left[\mathcal{A}(m(\ell))-\mathcal{A}\left(\ell^{*}\right)\right]+\int_{\ell^{*}}^{\ell}\left[\frac{1}{a(s)}\left(\varsigma_{3}+\left(\left(1+q_{1}\right) \varsigma_{2}\right)^{\alpha}-\varsigma_{3}\right)\right]^{1 / \alpha} \Delta s \\
& \leq-q_{1} \varsigma_{2}\left[\mathcal{A}(\ell)-\mathcal{A}\left(\ell^{*}\right)\right]+\left(1+q_{1}\right) \varsigma_{2}\left[\mathcal{A}(\ell)-\mathcal{A}\left(\ell^{*}\right)\right] \\
& \leq \varsigma_{2}\left[\mathcal{A}(\ell)-\mathcal{A}\left(\ell^{*}\right)\right]
\end{aligned}
$$

and

$$
\begin{aligned}
\left(\Gamma_{1} u_{1}\right)(\ell)+\left(\Gamma_{2} u_{2}\right)(\ell) & =-q(\ell) u_{1}(m(\ell))+\int_{\ell^{*}}^{\ell}\left[\frac{1}{a(s)}\left[\varsigma_{3}+\int_{s}^{\infty} \Lambda(\theta) u_{2}^{\beta}(\tau(\theta)) \Delta \theta\right]\right]^{1 / \alpha} \Delta s \\
& \geq\left[\int_{\ell^{*}}^{\ell} \varsigma_{3} \frac{1}{a(s)}\right]^{1 / \alpha} \Delta s \\
& =\varsigma_{3}^{1 / \alpha}\left[\mathcal{A}(\ell)-\mathcal{A}\left(\ell^{*}\right)\right] \\
& \geq \varsigma_{1}\left[\mathcal{A}(\ell)-\mathcal{A}\left(\ell^{*}\right)\right] .
\end{aligned}
$$

Therefore, $\Gamma_{1} u_{1}+\Gamma_{2} u_{2} \in \Omega_{\varsigma_{1}, \varsigma_{2}}$.

Next, we show that $\Gamma_{1}$ is a contraction mapping on $\Omega_{\zeta_{1}, \varsigma_{2}}$. Now for $u_{1}, u_{2} \in \Omega_{\zeta_{1}, \varsigma_{2}}$ and $\ell \geq \ell^{*}$, we have

$$
\left|\left(\Gamma_{1} u_{1}\right)(\ell)-\left(\Gamma_{1} u_{2}\right)(\ell)\right| \leq|q(\ell)|\left|u_{1}(m(\ell))-u_{2}(m(\ell))\right| \leq-q_{1}\left|u_{1}(m(\ell))-u_{2}(m(\ell))\right|,
$$

that is,

$$
\left\|\Gamma_{1} u_{1}-\Gamma_{1} u_{2}\right\| \leq-q_{1}\left\|u_{1}-u_{2}\right\| .
$$

Since $0 \leq-q_{1}<1, \Gamma_{1}$ is a contraction.

To show that $\Gamma_{2}$ is completely continuous, we will first show that $\Gamma_{2}$ is continuous. So fix $\ell \geq \ell^{*}$ and let $u_{k} \in \Omega_{\varsigma_{1}, \varsigma_{2}}$ be such that $u_{k}(\ell) \rightarrow u(\ell)$ as $k \rightarrow \infty$. By taking a subsequence if necessary and again calling it $u_{k}(\ell)$, we can assume that $u_{k}(\ell)-u(\ell)$ is of fixed sign, say $u_{k}(\ell) \geq u(\ell)$ for $k=1,2, \ldots$. Since $\Omega_{\varsigma_{1}, \varsigma_{2}}$ is closed, $u(\ell) \in \Omega_{\varsigma_{1}, \varsigma_{2}}$. By Lemma 1 with $\omega=1 / \alpha \leq 1$, we obtain 


$$
\begin{aligned}
\left|\left(\Gamma_{2} u_{k}\right)(\ell)-\left(\Gamma_{2} u\right)(\ell)\right|= & \mid \int_{\ell^{*}}^{\ell}\left[\frac{1}{a(s)}\left[\varsigma_{3}+\int_{s}^{\infty} \Lambda(\theta) u_{k}^{\beta}(\tau(\theta)) \Delta \theta\right]\right]^{1 / \alpha} \Delta s \\
& -\int_{\ell^{*}}^{\ell}\left[\frac{1}{a(s)}\left[\varsigma_{3}+\int_{s}^{\infty} \Lambda(\theta) u^{\beta}(\tau(\theta)) \Delta \theta\right]\right]^{1 / \alpha} \Delta s \mid \\
& \leq \int_{\ell^{*}}^{\ell}\left[\frac{1}{a(s)} \int_{s}^{\infty} \Lambda(\theta)\left|u_{k}^{\beta}(\tau(\theta))-u^{\beta}(\tau(\theta))\right| \Delta \theta\right]^{1 / \alpha} \Delta s .
\end{aligned}
$$

Since $\left|u_{k}^{\beta}(\tau(\theta))-u^{\beta}(\tau(\theta))\right| \rightarrow 0$ as $k \rightarrow \infty$, an application of Lebesgue's dominated convergence theorem shows that $\lim _{k \rightarrow \infty}\left|\left(\Gamma_{2} u_{k}\right)(\ell)-\left(\Gamma_{2} u\right)(\ell)\right| \rightarrow 0$, so $\Gamma_{2} u$ is continuous.

To show that $\Gamma_{2}$ is relatively compact, it suffices to show that the family of functions $\left\{\Gamma_{2} u: u \in \Omega_{\varsigma_{1}, \varsigma_{2}}\right\}$ is uniformly bounded and equicontinuous on $\left[\ell^{*}, \infty\right)_{\mathbb{T}}$. Clearly, $\Gamma_{2} u$ is uniformly bounded. To see that $\Gamma_{2}$ is equicontinuous, let $\epsilon>0$ be given and choose $\delta>0 \quad$ such that $\ell_{3}>\ell_{2} \geq \ell^{*} \quad$ and $\quad\left|\ell_{2}-\ell_{1}\right|<\delta \quad$ implies $\left|\mathcal{A}\left(\ell_{3}\right)-\mathcal{A}\left(\ell_{2}\right)\right|<\epsilon\left\{\frac{1}{\left[\left(1+q_{1}\right)_{5}\right]^{\alpha}-\varsigma_{3}}\right\}^{1 / \alpha}$. Then,

$$
\begin{aligned}
& \left|\left(\Gamma_{2} u\right)\left(\ell_{3}\right)-\left(\Gamma_{2} u\right)\left(\ell_{2}\right)\right| \\
& \quad=\left|\int_{\ell^{*}}^{\ell_{3}}\left[\frac{1}{a(s)} \int_{s}^{\infty} \Lambda(\theta) u^{\beta}(\tau(\theta)) \Delta \theta\right]^{1 / \alpha} \Delta s-\int_{\ell^{*}}^{\ell_{2}}\left[\frac{1}{a(s)} \int_{s}^{\infty} \Lambda(\theta) u^{\beta}(\tau(\theta)) \Delta \theta\right]^{1 / \alpha} \Delta s\right| \\
& \quad=\left|\int_{\ell_{2}}^{\ell_{3}}\left[\frac{1}{a(s)} \int_{s}^{\infty} \Lambda(\theta) u^{\beta}(\tau(\theta)) \Delta \theta\right]^{1 / \alpha} \Delta s\right| \\
& \quad \leq\left|\int_{\ell_{2}}^{\ell_{3}}\left[\frac{1}{a(s)} \int_{s}^{\infty} \Lambda(\theta) \varsigma_{2}^{\beta} \mathcal{A}^{\beta}(\tau(\theta)) \Delta \theta\right]^{1 / \alpha} \Delta s\right| \\
& \quad \leq\left[\mathcal{A}\left(\ell_{3}\right)-\mathcal{A}\left(\ell_{2}\right)\right]\left[\int_{s}^{\infty} \Lambda(\theta) \varsigma_{2}^{\beta} \mathcal{A}^{\beta}(\tau(\theta)) \Delta \theta\right]^{1 / \alpha} \Delta s<\epsilon .
\end{aligned}
$$

Thus, $\left\{\Gamma_{2} u: u \in \Omega_{\varsigma_{1} \varsigma_{2}}\right\}$ is uniformly bounded and equicontinuous on $\left[\ell^{*}, \infty\right)_{\mathbb{T}}$, and so $\Gamma_{2} u$ is relatively compact. By Krasnosel'skii's fixed point theorem [29, Lemma 5], $\Gamma_{1}+\Gamma_{2}$ has a unique fixed point $u \in \Omega_{\varsigma_{1}, \varsigma_{2}}$, i.e., $\Gamma_{1} u+\Gamma_{2} u=u$. That is,

$$
u(\ell)=-q(\ell) u(m(\ell))+\int_{\ell^{*}}^{\ell}\left[\frac{1}{a(s)}\left[\varsigma_{3}+\int_{s}^{\infty} \Lambda(\theta) u^{\beta}(\tau(\theta)) \Delta \theta\right]\right]^{1 / \alpha} \Delta s, \quad \ell \in\left[\ell^{*}, \infty\right) \mathbb{T} .
$$

is a nonoscillatory solution of (3).

Sufficiency: Now assume that $v$ is a nonoscillatory solution of (3). Then Lemma 3 holds for $\ell \in\left[\ell_{1}, \infty\right)_{\mathbb{T}}$ for some $\ell_{1} \geq \ell_{0}$, and there are two possible cases.

Case a Since $a\left(v^{\Delta}\right)^{\alpha}$ is nonincreasing and positive for $\ell \in\left[\ell_{1}, \infty\right)_{\mathbb{T}}$, we can find $\mathcal{C}>0$ and $\ell_{2}>\ell_{0}$ such that

$$
a(\ell)\left(v^{\Delta}(\ell)\right)^{\alpha} \leq \mathcal{C} \text { for } \ell \in\left[\ell_{2}, \infty\right)_{\mathbb{T}} .
$$

Integrating from $\ell_{2}$ to $\ell$ gives 


$$
v(\ell) \leq v\left(\ell_{2}\right)+\mathcal{C}^{1 / \alpha} \int_{\ell_{2}}^{\ell} \frac{\Delta s}{a^{1 / \alpha}(s)}=v\left(\ell_{2}\right)+\mathcal{C}^{1 / \alpha}\left(\mathcal{A}(\ell)-\mathcal{A}\left(\ell_{2}\right)\right) .
$$

Since $\lim _{\ell \rightarrow \infty} \mathcal{A}(\ell)=\infty$,

$$
v(\ell) \leq \mathcal{C}^{1 / \alpha} \mathcal{A}(\ell)
$$

for $\ell$ sufficiently large, say $\ell \geq \ell_{3}$. Now $\beta<\gamma$ and (6) imply

$$
v^{\beta}(\tau(\ell))=v^{\beta-\gamma}(\tau(\ell)) v^{\gamma}(\tau(\ell)) \geq\left[\mathcal{C}^{1 / \alpha} \mathcal{A}(\tau(\ell))\right]^{\beta-\gamma} v^{\gamma}(\tau(\ell)) .
$$

Therefore, (3) becomes

$$
\left[a(t)\left(v^{\Delta}(\ell)\right)^{\alpha}\right]^{\Delta}+\Lambda(\ell)\left[\mathcal{C}^{1 / \alpha} \mathcal{A}(\tau(\ell))\right]^{\beta-\gamma} v^{\gamma}(\tau(\ell)) \leq 0 .
$$

Integrating the last inequality from $\ell \geq \ell_{3}$ to $\infty$ gives

$$
\lim _{t \rightarrow \infty} a(t)\left(v^{\Delta}(t)\right)^{\alpha}-a(\ell)\left(v^{\Delta}(\ell)\right)^{\alpha}+\int_{\ell}^{\infty} \Lambda(s)\left[\mathcal{C}^{1 / \alpha} \mathcal{A}(\tau(s))\right]^{\beta-\gamma} v^{\gamma}(\tau(s)) \Delta s \leq 0,
$$

which implies

$$
a(\ell)\left(v^{\Delta}(\ell)\right)^{\alpha} \geq \int_{\ell}^{\infty} \Lambda(s)\left[\mathcal{C}^{1 / \alpha} \mathcal{A}(\tau(s))\right]^{\beta-\gamma} \nu^{\gamma}(\tau(s)) \Delta s .
$$

As a result,

$$
v^{\Delta}(\ell) \geq\left[\frac{1}{a(\ell)} \int_{\ell}^{\infty} \Lambda(s)\left[\mathcal{C}^{1 / \alpha} \mathcal{A}(\tau(s))\right]^{\beta-\gamma} v^{\gamma}(\tau(s)) \Delta s\right]^{1 / \alpha} .
$$

Integrating this from $\ell_{3}$ to $\ell$, we have

$$
v(\ell) \geq \int_{\ell_{3}}^{\ell}\left[\frac{1}{a(s)} \int_{s}^{\infty} \Lambda(\theta)\left[\mathcal{C}^{1 / \alpha} \mathcal{A}(\tau(\theta))\right]^{\beta-\gamma} v^{\gamma}(\tau(\theta)) \Delta \theta\right]^{1 / \alpha} \Delta s .
$$

Consequently,

$$
v(\ell) \geq\left[\mathcal{A}(\ell)-\mathcal{A}\left(\ell_{1}\right)\right]\left[\int_{s}^{\infty} \Lambda(\theta)\left[\mathcal{C}^{1 / \alpha} \mathcal{A}(\tau(\theta))\right]^{\beta-\gamma} v^{\gamma}(\tau(\theta)) \Delta \theta\right]^{1 / \alpha} .
$$

Clearly, $\int_{\ell_{3}}^{\ell} \frac{1}{a(s)} \Delta s=\mathcal{A}(\ell)-\mathcal{A}\left(\ell_{3}\right)=\pi(\ell) \mathcal{A}(\ell)$, where $\pi(\ell)=\frac{\mathcal{A}(\ell)-\mathcal{A}\left(\ell_{3}\right)}{\mathcal{A}(\ell)}$. In view of $\left(\mathcal{H}_{3}\right)$, we have $\lim _{\ell \rightarrow \infty} \pi(\ell)=1$, so there exists $\ell_{4} \geq \ell_{3}$ and $\pi^{*} \in(0,1)$ such that $\pi(\ell) \geq \pi^{*}$, that is,

$$
\mathcal{A}(\ell)-\mathcal{A}\left(\ell_{3}\right) \geq \pi^{*} \mathcal{A}(\ell) \text { for } \ell \in\left[\ell_{4}, \infty\right)_{\mathbb{T}} .
$$

\section{Setting}

$$
\Psi(\ell)=\int_{\ell}^{\infty} \Lambda(s)\left(\mathcal{C}^{1 / \alpha} \mathcal{A}(\tau(s))^{\beta-\gamma} v^{\gamma}(\tau(s)) \Delta s,\right.
$$

in (8), we have 


$$
v(\ell) \geq\left[\mathcal{A}(\ell)-\mathcal{A}\left(\ell_{1}\right)\right] \Psi^{1 / \alpha}(\ell) .
$$

and in view of (9),

$$
v(\ell) \geq \pi^{*} \mathcal{A}(\ell) \Psi^{1 / \alpha}(\ell)
$$

for $\ell \in\left[\ell_{4}, \infty\right)_{\mathbb{T}}$. From the preceding inequality, it is easy to verify that

$$
\frac{v^{\gamma}(\ell)}{\mathcal{C}^{\gamma / \alpha} \mathcal{A}^{\gamma}(\ell)} \geq\left(\frac{\pi^{*}}{\mathcal{C}^{1 / \alpha}}\right)^{\gamma} \Psi^{\gamma / \alpha}(\ell)
$$

which implies that

$$
\frac{\nu^{\gamma}(\tau(\ell))}{\mathcal{C}^{\gamma / \alpha} \mathcal{A}^{\gamma}(\tau(\ell))} \geq\left(\frac{\pi^{*}}{\mathcal{C}^{1 / \alpha}}\right)^{\gamma} \Psi^{\gamma / \alpha}(\tau(\ell))
$$

for $\ell \in\left[\ell_{5}, \infty\right)_{\mathbb{T}} \subset\left[\ell_{4}, \infty\right)_{\mathbb{T}}$. From (10), we have

$$
\begin{aligned}
\Psi^{\Delta}(\ell) & =\left(\int_{\ell}^{\infty} \Lambda(s)\left(\mathcal{C}^{1 / \alpha} \mathcal{A}(\tau(s))^{\beta-\gamma} v^{\gamma}(\tau(s)) \Delta s\right)^{\Delta}\right. \\
& =-\Lambda(\ell)\left(\mathcal{C}^{1 / \alpha} \mathcal{A}(\tau(\ell))^{\beta-\gamma} v^{\gamma}(\tau(\ell))\right. \\
& =-\Lambda(\ell)\left(\mathcal{C}^{1 / \alpha} \mathcal{A}(\tau(\ell))^{\beta}\left(\frac{v(\tau(\ell))}{\mathcal{C}^{1 / \alpha} \mathcal{A}(\tau(\ell))}\right)^{\gamma}\right. \\
& \leq-\left(\pi^{*}\right)^{\gamma} \mathcal{C}^{(\beta-\gamma) / \alpha} \Lambda(\ell) \mathcal{A}^{\beta}(\tau(\ell)) \Psi^{\gamma / \alpha}(\tau(\ell)) .
\end{aligned}
$$

From Lemma 2 with $\omega=\gamma / \alpha$ and $x=\Psi(\ell)$ and the fact that $\gamma<\alpha$, it follows that

$$
\begin{aligned}
-\left[\Psi^{1-\gamma / \alpha}(\ell)\right]^{\Delta} & \geq-(1-\gamma / \alpha) \Psi^{-\gamma / \alpha}(\ell) \Psi^{\Delta}(\ell) \\
& \geq\left(\pi^{*}\right)^{\gamma} \mathcal{C}^{(\beta-\gamma) / \alpha}(1-\gamma / \alpha) \Psi^{-\gamma / \alpha}(\ell) \Lambda(\ell) \mathcal{A}^{\beta}(\tau(\ell)) \Psi^{\gamma / \alpha}(\tau(\ell)) \\
& =\left(\pi^{*}\right)^{\gamma} \mathcal{C}^{(\beta-\gamma) / \alpha}(1-\gamma / \alpha) \Lambda(\ell) \mathcal{A}^{\beta}(\tau(\ell))
\end{aligned}
$$

for $\ell \in\left[\ell_{5}, \infty\right)_{\mathbb{T}}$. Integrating (11) from $\ell_{5}$ to $\ell$,

$$
-\Psi^{1-\gamma / \alpha}(\ell)+\Psi^{1-\gamma / \alpha}\left(\ell_{5}\right) \geq\left(\pi^{*}\right)^{\gamma} \mathcal{C}^{(\beta-\gamma) / \alpha}(1-\gamma / \alpha) \int_{\ell_{5}}^{\ell} \Lambda(s) \mathcal{A}^{\beta}(\tau(s)) \Delta s
$$

so

$$
\int_{\ell_{5}}^{\ell} \Lambda(s) \mathcal{A}^{\beta}(\tau(s)) \Delta s \leq \frac{\mathcal{C}^{(\gamma-\beta) / \alpha}}{\left(\pi^{*}\right)^{\gamma}(1-\gamma / \alpha)} \Psi^{1-\gamma / \alpha}\left(\ell_{5}\right)
$$

contradicting $\left(\mathcal{H}_{4}\right)$.

Case $\mathbf{b}$ Now suppose $v<0$ for $\ell \in\left[\ell_{0}, \infty\right)_{\mathbb{T}}$. Then $u(\ell) \rightarrow 0$ as $\ell \rightarrow \infty$ by Lemma 4 . This completes the proof of the theorem.

The following corollary is immediate. 
Corollary 6 Under the assumption of Theorem 5, every unbounded solution of (3) oscillates if and only if $\left(\mathcal{H}_{4}\right)$ holds.

Theorem 7 Let $\left(\mathcal{H}_{1}\right)-\left(\mathcal{H}_{3}\right)$ hold, $\sigma(\tau(\ell))=\tau(\sigma(\ell)), a^{\Delta}(\ell) \geq 0$, and there is a constant $\gamma \in \mathbb{R}_{+}$such that $\alpha<\gamma<\beta$. Then any solution $u(\ell)$ of (3) is either oscillatory or satisfies $\lim _{\ell \rightarrow \infty} u(\ell)=0$ if and only if

$\left(\mathcal{H}_{5}\right) \quad \lim _{\ell \rightarrow \infty} \int_{\ell_{0}}^{\ell} \int_{s}^{\infty}\left(\frac{\Lambda(\theta)}{a(s)}\right)^{1 / \alpha} \Delta \theta \Delta s=\infty$.

Proof Necessity: Assume that $\left(\mathcal{H}_{5}\right)$ does not hold so that there exists $\ell_{1}>\ell_{0}$ such that

$$
\int_{\ell_{1}}^{\infty}\left[\frac{1}{a(s)} \int_{s}^{\infty} \Lambda(\theta) \Delta \theta\right]^{1 / \alpha} \Delta s<\infty .
$$

Letting

$$
\chi=\left\{u: u \in C_{r d}\left(\left[\ell_{0}, \infty\right)_{\mathbb{T}}, \mathbb{R}\right) \mid \sup _{\ell \in\left[\ell_{0}, \infty\right)_{\mathbb{T}}} u(\ell)<\infty\right\},
$$

we see that $\chi$ is a Banach space with the norm $\|u\|=\sup _{\ell \in\left[\ell_{0}, \infty\right)_{\mathbb{T}}} u(\ell)$. Choose $\varsigma_{1}>0$ and $\varsigma_{2}>0$ so that $\varsigma_{1}-q_{1} \varsigma_{2}<\varsigma_{2}$ and consider $\Omega_{\varsigma_{1}, \varsigma_{2}} \subset \chi$ to be

$$
\Omega_{\varsigma_{1}, \varsigma_{2}}=\left\{u \in \chi: \varsigma_{1} \leq u(\ell) \leq \varsigma_{2}, \quad \ell \in\left[\ell_{0}, \infty\right)_{\mathbb{T}}\right\} .
$$

By (12), we can find $\ell^{*}>\ell_{1}$ and $\varsigma_{3}>0$ such that $\varsigma_{1}<\varsigma_{3}<\left(1+q_{1}\right) \varsigma_{2}$ and

$$
\int_{\ell^{*}}^{\infty}\left[\frac{1}{a(s)} \int_{s}^{\infty} \Lambda(\theta) \Delta \theta\right]^{1 / \alpha} \Delta s \leq \frac{\left(1+q_{1}\right) \varsigma_{2}-\varsigma_{3}}{\varsigma_{2}^{\beta / \alpha}} .
$$

Define two maps $\Gamma_{1}$ and $\Gamma_{2}$ on $\Omega$ by

$$
\left(\Gamma_{1} u\right)(\ell)= \begin{cases}\left(\Gamma_{1} u\right)\left(\ell^{*}\right), & \ell \in\left[\ell_{0}, \ell^{*}\right)_{\mathbb{T}}, \\ \varsigma_{3}-q(\ell) u(m(\ell)), & \ell \in\left[\ell^{*}, \infty\right)_{\mathbb{T}}\end{cases}
$$

and

$$
\left(\Gamma_{2} u\right)(\ell)= \begin{cases}\left(\Gamma_{2} u\right)\left(\ell^{*}\right), & \ell \in\left[\ell_{0}, \ell^{*}\right)_{\mathbb{T}}, \\ \int_{\ell^{*}}^{\ell}\left[\frac{1}{a(s)} \int_{s}^{\infty} \Lambda(\theta) u^{\beta}(\tau(\theta)) \Delta \theta\right]^{1 / \alpha} \Delta s, & \ell \in\left[\ell^{*}, \infty\right)_{\mathbb{T}} .\end{cases}
$$

To show that $\Gamma_{1}+\Gamma_{2}: \Omega \rightarrow \Omega$, let $u_{1}, u_{2} \in \Omega$. Then from (13),

$$
\begin{aligned}
\left(\Gamma_{1} u_{1}\right)(\ell)+\left(\Gamma_{2} u_{2}\right)(\ell) & =\varsigma_{3}-q(\ell) u_{1}(m(\ell))+\int_{\ell^{*}}^{\ell}\left[\frac{1}{a(s)} \int_{s}^{\infty} \Lambda(\theta) u_{2}^{\beta}(\tau(\theta)) \Delta \theta\right]^{1 / \alpha} \Delta s \\
& \leq \varsigma_{3}-q_{1} \varsigma_{2}+\int_{\ell^{*}}^{\ell}\left[\frac{1}{a(s)} \int_{s}^{\infty} \Lambda(\theta) u_{2}^{\beta}(\tau(\theta)) \Delta \theta\right]^{1 / \alpha} \Delta s \\
& \leq \varsigma_{3}-q_{1} \varsigma_{2}+\varsigma_{2}^{\beta / \alpha} \int_{\ell^{*}}^{\ell}\left[\frac{1}{a(s)} \int_{s}^{\infty} \Lambda(\theta) \Delta \theta\right]^{1 / \alpha} \Delta s \\
& \leq \varsigma_{2}
\end{aligned}
$$


and

$$
\left(\Gamma_{1} u_{1}\right)(\ell)+\left(\Gamma_{2} u_{2}\right)(\ell) \geq \varsigma_{3}-q(\ell) u_{1}(m(\ell)) \geq \varsigma_{3} \geq \varsigma_{1}
$$

for $\ell \geq \ell^{*}$. Hence, $\Gamma_{1} u_{1}+\Gamma_{2} u_{2} \in \Omega_{\varsigma_{1}, \varsigma_{2}}$.

To see that $\Gamma_{1}$ is a contraction, let $u_{1}, u_{2} \in \Omega_{\varsigma_{1}, \varsigma_{2}}$ and $\ell \geq \ell^{*}$. Then,

$$
\left|\left(\Gamma_{1} u_{1}\right)(\ell)-\left(\Gamma_{1} u_{2}\right)(\ell)\right| \leq|q(\ell)|\left|u_{1}(m(\ell))-u_{2}(m(\ell))\right| \leq-q_{1}\left|u_{1}(m(\ell))-u_{2}(m(\ell))\right|,
$$

so

$$
\left\|\Gamma_{1} u_{1}-\Gamma_{1} u_{2}\right\| \leq-q_{1}\left\|u_{1}-u_{2}\right\|
$$

i.e., $\Gamma_{1}$ is a contraction mapping.

To show that $\Gamma_{2}$ is completely continuous, we begin by letting $u_{k} \in \Omega_{\varsigma_{1}, \varsigma_{2}}$ be such that $u_{k}(\ell) \rightarrow u(\ell)$ as $k \rightarrow \infty$. Since $\Omega_{\varsigma_{1}, \varsigma_{2}}$ is closed, $u(\ell) \in \Omega_{\varsigma_{1}, \varsigma_{2}}$. Now

$$
\left|\left(\Gamma_{2} u_{k}\right)(\ell)-\left(\Gamma_{2} u\right)(\ell)\right| \leq \int_{\ell^{*}}^{\ell}\left[\frac{1}{a(s)} \int_{s}^{\infty} \Lambda(\theta)\left|u_{k}^{\beta}(\tau(\theta))-u^{\beta}(\tau(\theta))\right| \Delta \theta\right]^{1 / \alpha} \Delta s .
$$

Since $\left|u_{k}^{\beta}(\tau(\theta))-u^{\beta}(\tau(\theta))\right| \rightarrow 0$ as $k \rightarrow \infty$, an application of Lebesgue's dominated convergence theorem implies $\lim _{k \rightarrow \infty}\left|\left(\Gamma_{2} u_{k}\right)(\ell)-\left(\Gamma_{2} u\right)(\ell)\right| \rightarrow 0$. Hence, $\Gamma_{2} u$ is continuous. To show that $\Gamma_{2} u$ is relatively compact, it suffices to show that the family of functions $\left\{\Gamma_{2} u: u \in \Omega_{\varsigma_{1}, \varsigma_{2}}\right\}$ is uniformly bounded and equicontinuous on $\left[\ell^{*}, \infty\right)_{\mathbb{T}}$. The uniform boundedness is clear.

To show $\Gamma_{2} u$ is equicontinuous, let $\epsilon>0$ be given and choose $\delta>0$ such that $\ell_{3}>\ell_{2} \geq \ell^{*}$ and $\left|\ell_{2}-\ell_{1}\right|<\delta$ implies

$$
\int_{\ell_{2}}^{\ell_{3}}\left[\frac{1}{a(s)} \int_{s}^{\infty} \Lambda(\theta) \Delta \theta\right]^{1 / \alpha} \Delta s<\frac{\epsilon}{\varsigma_{2}^{\beta / \alpha}}
$$

Then,

$$
\begin{aligned}
& \left|\left(\Gamma_{2} u\right)\left(\ell_{3}\right)-\left(\Gamma_{2} u\right)\left(\ell_{2}\right)\right| \\
& \quad=\left|\int_{\ell^{*}}^{\ell_{3}}\left[\frac{1}{a(s)} \int_{s}^{\infty} \Lambda(\theta) u^{\beta}(\tau(\theta)) \Delta \theta\right]^{1 / \alpha} \Delta s-\int_{\ell^{*}}^{\ell_{2}}\left[\frac{1}{a(s)} \int_{s}^{\infty} \Lambda(\theta) u^{\beta}(\tau(\theta)) \Delta \theta\right]^{1 / \alpha} \Delta s\right| \\
& \quad=\left|\int_{\ell_{2}}^{\ell_{3}}\left[\frac{1}{a(s)} \int_{s}^{\infty} \Lambda(\theta) u^{\beta}(\tau(\theta)) \Delta \theta\right]^{1 / \alpha} \Delta s\right| \\
& \quad \leq \varsigma_{2}^{\beta / \alpha} \int_{\ell_{2}}^{\ell_{3}}\left[\frac{1}{a(s)} \int_{s}^{\infty} \Lambda(\theta) \Delta \theta\right]^{1 / \alpha} \Delta s<\epsilon .
\end{aligned}
$$

Therefore, $\Gamma_{2} u$ is relatively compact, and by Krasnosel'skii's fixed point theorem [, Lemma 5], $29 \Gamma_{1}+\Gamma_{2}$ has a unique fixed point $u \in \Omega_{\varsigma_{1}, \varsigma_{2}}$. It follows that

$$
u(\ell)=\varsigma_{3}-q(\ell) u(m(\ell))+\int_{\ell^{*}}^{\ell}\left[\frac{1}{a(s)} \int_{s}^{\infty} \Lambda(\theta) u^{\beta}(\tau(\theta)) \Delta \theta\right]^{1 / \alpha} \Delta s, \ell \in\left[\ell^{*}, \infty\right)_{\mathbb{T}}
$$


is a nonoscillatory solution of (3).

Sufficiency: Let $u$ be a nonoscillatory solution of (3) with Lemma 3 holding for $\ell \in\left[\ell_{1}, \infty\right)_{\mathbb{T}}$. We again consider two cases.

Case a Let $v>0$; then $u(\ell) \geq v(\ell)$ for $\ell \in\left[\ell_{1}, \infty\right)_{\mathbb{T}}$. From the fact that $v^{\Delta}(\ell)>0$ for $\ell \in\left[\ell_{1}, \infty\right)_{\mathbb{T}}$, it follows that $v(\tau(\ell)) \geq v\left(\tau\left(\ell_{1}\right)\right)=\mathcal{C}$ for $\ell \in\left[\ell_{2}, \infty\right)_{\mathbb{T}}$ for some $\ell_{2} \geq \ell_{1}$. Since $\gamma<\beta$,

$$
v^{\beta}(\tau(\ell))=v^{\beta-\gamma}(\tau(\ell)) v^{\gamma}(\tau(\ell)) \geq \mathcal{C}^{\beta-\gamma} v^{\gamma}(\tau(\ell)) .
$$

Using (14) in (3), we obtain

$$
\left[a(\ell)\left(v^{\Delta}(\ell)\right)^{\alpha}\right]^{\Delta}+\mathcal{C}^{\beta-\gamma} \Lambda(\ell) v^{\gamma}(\tau(\ell)) \leq 0,
$$

and an integration from $\ell$ to $\infty$ gives

$$
\lim _{t \rightarrow \infty} a(t)\left(v^{\Delta}(t)\right)^{\alpha}-a(\ell)\left(v^{\Delta}(\ell)\right)^{\alpha}+\mathcal{C}^{\beta-\gamma} \int_{\ell}^{\infty} \Lambda(s) v^{\gamma}(\tau(s)) \Delta s \leq 0,
$$

that is,

$$
\mathcal{C}^{\beta-\gamma} \int_{\ell}^{\infty} \Lambda(s) v^{\gamma}(\tau(s)) \Delta s \leq a(\ell)\left(v^{\Delta}(\ell)\right)^{\alpha} \leq a(\tau(\ell))\left(v^{\Delta}(\tau(\ell))\right)^{\alpha} .
$$

Using the fact that $a^{\Delta}(\ell) \geq 0$, we see that

$$
\left(v^{\Delta}(\tau(\ell))\right)^{\alpha} \geq \frac{\mathcal{C}^{\beta-\gamma}}{a(\ell)} \int_{\ell}^{\infty} \Lambda(s) v^{\gamma}(\tau(s)) \Delta s,
$$

which implies

$$
\begin{aligned}
v^{\Delta}(\tau(\ell)) & \geq \frac{\mathcal{C}^{(\beta-\gamma) / \alpha}}{a^{1 / \alpha}(\ell)}\left[\int_{\ell}^{\infty} \Lambda(s) v^{\gamma}(\tau(s)) \Delta s\right]^{1 / \alpha} \\
& \geq \frac{\mathcal{C}^{(\beta-\gamma) / \alpha}}{a^{1 / \alpha}(\ell)}\left[\int_{\sigma(\ell)}^{\infty} \Lambda(s) v^{\gamma}(\tau(s)) \Delta s\right]^{1 / \alpha} \\
& \geq \frac{\mathcal{C}^{(\beta-\gamma) / \alpha}}{a^{1 / \alpha}(\ell)}\left[\int_{\sigma(\ell)}^{\infty} \Lambda(s) \Delta s\right]^{1 / \alpha}\left(v^{\sigma}(\tau(\ell))\right)^{\gamma / \alpha},
\end{aligned}
$$

that is

$$
v^{\Delta}(\tau(\ell))\left(v^{\sigma}(\tau(\ell))\right)^{-\gamma / \alpha} \geq \frac{\mathcal{C}^{(\beta-\gamma) / \alpha}}{a^{1 / \alpha}(\ell)}\left[\int_{\sigma(\ell)}^{\infty} \Lambda(s) \Delta s\right]^{1 / \alpha} .
$$

Since $\alpha<\gamma$, by Lemma 2

$$
\frac{\mathcal{C}^{(\beta-\gamma) / \alpha}}{a^{1 / \alpha}(\ell)}\left[\int_{\sigma(\ell)}^{\infty} \Lambda(s) \Delta s\right]^{1 / \alpha} \leq v^{\Delta}(\tau(\ell))\left(v^{\sigma}(\tau(\ell))\right)^{-\gamma / \alpha} \leq \frac{\left[v^{1-\gamma / \alpha}(\tau(\ell))\right]^{\Delta}}{1-\gamma / \alpha} .
$$

Integrating the preceding inequality from $\ell_{2}$ to $\ell$ gives 


$$
\begin{aligned}
\mathcal{C}^{(\beta-\gamma) / \alpha} \int_{\ell_{2}}^{\ell}\left[\frac{1}{a(s)} \int_{s}^{\infty} \Lambda(\theta) \Delta \theta\right]^{1 / \alpha} \Delta s & \left.\leq \frac{1}{1-\gamma / \alpha} \int_{\ell_{2}}^{\ell}\left[v^{1-\gamma / \alpha}(\tau(s))\right)\right]^{\Delta} \Delta s \\
& \left.=\frac{1}{\gamma / \alpha-1}\left[v^{1-\gamma / \alpha}\left(\tau\left(\ell_{2}\right)\right)\right)-v^{1-\gamma / \alpha}(\tau(\ell))\right] \\
& \leq \frac{1}{\gamma / \alpha-1} v^{1-\gamma / \alpha}\left(\tau\left(\ell_{2}\right)\right),
\end{aligned}
$$

contradicting $\left(\mathcal{H}_{5}\right)$.

Case $\mathbf{b}$ If $v<0$, then $u(\ell) \rightarrow 0$ by Lemma 4 . This proves the theorem.

The following corollary is analogous to Corollary 6.

Corollary 8 Under the assumption of Theorem 5, every unbounded solution of (3) oscillates if and only if $\left(\mathcal{H}_{5}\right)$ holds.

\section{Discussion}

First, we constructed an appropriate Banach space as the setting on which to defining two mappings $\Gamma_{1}$ and $\Gamma_{2}$. The sum of these two mappings is an operator that is equivalent to an integral representation of the solution to the nonlinear dynamic equation (3) under investigation. By applying Krasnosel'skii's fixed point theorem on time scales, it was then possible to obtain a fixed point of the operator that in turn corresponds to a solution of Eq. (3). Once this was accomplished, various results on the qualitative properties of solution were obtained. For example, we found sufficient conditions for positive solutions to converge to zero (Lemma 4). In addition, we were able to prove necessary and sufficient conditions for a solution to either oscillate or converge to zero (Theorems 5 and 7 ), and necessary and sufficient conditions for unbounded solutions to oscillate (Corollaries 6 and 8).

\section{Conclusion}

In this work, we discuss two classes of oscillation criteria for (3). Note that Theorem 5 and Theorem 7 guarantee that a solution of (3) either oscillates or converges to zero. In Corollaries 6 and 8, we restrict the solutions to make (3) oscillatory. Here, we formulate some interesting problem for future research:

1. Is it possible to find necessary and sufficient conditions for the oscillation of

$$
\left[a(\ell)\left((u(\ell)+q(\ell) u(m(\ell)))^{\Delta}\right)^{\alpha}\right]^{\Delta}+\Lambda(\ell) u^{\beta}([\tau(\ell)])=0
$$

under the assumption $\beta<\gamma<\alpha$ or $\alpha<\gamma<\beta$ ?

2. Following the work in $[19,21]$, is it possible to find necessary and sufficient conditions for the oscillation of the forced equation

$$
\left[a(\ell)\left((u(\ell)+q(\ell) u(m(\ell)))^{\Delta}\right)^{\alpha}\right]^{\Delta}+\Lambda(\ell) u^{\beta}(\tau(\ell))=f(\ell)
$$

with either $\beta<\gamma<\alpha$ or $\alpha<\gamma<\beta$ ? 


\section{Authors' contributions}

Each of the authors contributed equally to the research, writing, and preparation of this manuscript. In particular, GC, SG, and JG participated in the writing and revising of the manuscript. The formulation of the results was a joint effort by GC, SG, and JG. Although JG is serving as the corresponding author, GC and SG approved the final version of the paper before it was submitted. All authors read and approved the final manuscript.

\section{Funding}

There are no funding sources to report.

\section{Availability of data and materials}

Data sharing is not applicable to this article as no datasets were generated or analyzed during the current study.

\section{Declarations}

\section{Competing interests}

The authors declare that they have no competing interests.

\section{Author details}

${ }^{1}$ Department of Mathematics, Sambalpur University, Sambalpur 768019, India. ${ }^{2}$ Department of Engineering Mathematics, Faculty of Engineering, Cairo University, Orman, Giza 12221, Egypt. ${ }^{3}$ Department of Mathematics, University of Tennessee at Chattanooga, Chattanooga, TN 37403, USA.

Received: 24 March 2021 Accepted: 10 October 2021

Published online: 30 October 2021

\section{References}

1. Agarwal, R.P., Grace, S.R., O'Regan, D.: Oscillation Theory for Second Order Linear. Half-Linear, Superlinear and Sublinear Dynamic Equations, Kluwer, Dordrecht (2002)

2. Agarwal, R.P., Bohner, M., Grace, S.R.: Discrete Oscillation Theory. Hindawi, New York (2005)

3. Agarwal, R.P., O'Regan, D., Saker, S.H.: Oscillation criteria for second-order nonlinear neutral delay dynamic equations. J. Math. Anal. Appl. 300, 203-217 (2004)

4. Boe, E., Chang, H.C.: Dynamics of delayed systems under feedback control. Chem. Eng. Sci. 44, 1281-1294 (1989)

5. Bohner, M., Peterson, A.: Dynamic Equations on Time Scales: An Introduction with Applications. Birkhäuser, Boston (2001)

6. Bohner, M., Peterson, A.: Advances in Dynamic Equations on Time Scales. Birkhäuser, Boston (2003)

7. Bohner, M., Li, T.: Oscillation of second order $p$-Laplace dynamic equations with a nonpositive neutral coefficient. Appl. Math. Lett. 37, 72-76 (2014)

8. Chen, D.X:: Oscillation of second-order Emden-Fowler neutral delay dynamic equations on time scales. Math. Comput. Model. 51, 1221-1229 (2010)

9. Deng, X.H., Wang, Q.R.: Oscillation and nonoscillation for second order nonlinear neutral functional dynamic equations on time scales. Electron. J. Differ. Equ. 2013(234), 1-17 (2013)

10. Deng, X.H., Wang, Q.R., Zhou, Z.: Generalized Philos-type oscillation criteria for second order nonlinear neutral delay dynamic equations on time scales. Appl. Math. Lett. 57, 69-76 (2016)

11. Dosly, O., Hilger, S.: A necessary and sufficient condition for oscillation of the Sturm-Liouville dynamic equation on time scales. J. Comput. Appl. Math. 141, 147-158 (2002)

12. Driver, D.R.: A mixed neutral system. Nonlinear Anal. 8, 155-158 (1984)

13. Erbe, L., Baoguo, J., Mert, R.: A Wong-type necessary and sufficient condition for nonoscillation of second order linear dynamic equations on time scales. Commun. Appl. Anal. 18, 41-58 (2014)

14. Erbe, L., Hassan, T.S., Peterson, A.: Oscillation criteria for nonlinear functional neutral dynamic equations on time scales. J. Differ. Equ. Appl. 15, 1097-1116 (2009)

15. Grace, S.R., Agarwal, R.P., Bohner, M., O'Regan, D.: Oscillation of second order strongly superlinear and strongly sublinear dynamic equations. Commun. Nonlinear Sci. Numer. Simul. 14, 3463-3471 (2009)

16. Hale, J.K.: Theory of Functional Differential Equations. Springer, New York (1977)

17. Hilger, S.: Analysis on measure chain-a unified approach to continuous and discrete calculus. Results Math. 18 $18-56(1990)$

18. Karpuz, B.: Sufficient conditions for the oscillation and asymptotic behaviour of higher-order dynamic equations of neutral type. Appl. Math. Comput. 221, 453-462 (2013)

19. Karpuz, B.: Necessary and sufficient conditions on the asymptotic behavior of second-order neutral delay dynamic equations with positive and negative coefficients. Math. Methods Appl. Sci. 37, 1219-1231 (2014)

20. Li, Q., Wang, R., Chen, F.: Oscillation of second-order nonlinear delay differential equations with nonpositive neutral coefficients. Adv. Differ. Equ. 2015(35), 1-7 (2015)

21. Mert, R., Zafer, A.: A necessary and sufficient condition for oscillation of second order sublinear delay dynamic equations. Discrete Contin. Dyn. Syst. 2011, 1061-1067 (2011)

22. Meng, F., Zheng, Z.: Recent development in oscillatory properties of certain differential equations. J. Appl. Anal. Comput. 8, 1282-1306 (2018) 
23. Saker, S.H., O'Regan, D.: New oscillation criteria for second-order neutral functional dynamic equations via the generalized Riccati substitution. Commun. Nonlinear Sci. Numer. Simulat. 16, 423-434 (2011)

24. Shi, Y., Han, Z., Sun, Y.: Oscillation criteria for a generalized Emden-Fowler dynamic equation on time scales. Adv. Differ. Equ. 2016(3), 1-12 (2016)

25. Tripathy, A.K.: Some oscillation results for second order nonlinear dynamic equations of neutral type. Nonlinear Anal. 71, e1727-e1735 (2009)

26. Yang, Q.S., Xu, Z:: Oscillation criteria for second order quasilinear neutral delay differential equations on time scales. Comput. Math. Appl. 62, 3682-3691 (2011)

27. Yang, Q.S., Xu, Z., Long, P.: Oscillation criteria for quasi-linear neutral delay dynamic equations on time scales. Math. Slovaca 66, 185-196 (2016)

28. Zhang, M., Chen, W., El-Sheikh, M.M.A., Sallam, R.A., Hassan, A.M., Li, T.: Oscillation criteria for second-order nonlinear delay dynamic equations of neutral type. Adv. Differ. Equ. 2018(26), 1-9 (2018)

29. Zhu, Z.Q., Wang, Q.R.: Existence of nonoscillatory solutions to neutral dynamic equations on time scales. J. Math. Anal. Appl. 335, 751-762 (2007)

\section{Publisher's Note}

Springer Nature remains neutral with regard to jurisdictional claims in published maps and institutional affiliations.

\section{Submit your manuscript to a SpringerOpen ${ }^{\odot}$ journal and benefit from:}

- Convenient online submission

- Rigorous peer review

- Open access: articles freely available online

- High visibility within the field

- Retaining the copyright to your article

Submit your next manuscript at $\boldsymbol{\Delta}$ springeropen.com 